\title{
PAPER
}

\section{The impact of culture on physiological processes of emotion regulation: a comparison of US and Chinese preschoolers}

\section{Adam S. Grabell, ${ }^{1}$ Sheryl L. Olson, ${ }^{1}$ Alison L. Miller, ${ }^{1}$ Daniel A. Kessler, ${ }^{1}$ Barbara Felt, ${ }^{1}$ Niko Kaciroti, ${ }^{1}$ Li Wang ${ }^{2}$ and Twila Tardif ${ }^{1}$}

1. University of Michigan, Ann Arbor, Michigan, USA

2. Peking University, Beijing, China

\begin{abstract}
Cognitive determinants of emotion regulation, such as effortful control, have been hypothesized to modulate young children's physiological response to emotional stress. It is unknown, however, whether this model of emotion regulation generalizes across Western and non-Western cultures. The current study examined the relation between both behavioral and questionnaire measures of effortful control and densely sampled, stress-induced cortisol trajectories in U.S. and Chinese preschoolers. Participants were 3- to 5-year-old children recruited from the United States $(\mathrm{N}=57)$ and Beijing, China $(\mathrm{N}=60)$. Consistent with our hypothesis, U.S. children showed a significant negative relation between maternal-rated inhibitory control and both cortisol reactivity and recovery. However, this was not replicated in the Chinese sample. Children in China showed a significant positive relation between maternal-rated attentional focusing and cortisol reactivity that was not seen in the U.S. Results suggest that children who reside in Western and non-Western cultures have different predictors of their emotion-related stress response.
\end{abstract}

\section{Research highlights}

- This is the first study to examine a complex model of emotion regulation in Western and non-Western preschool children.

- The methodology of current study is unique for this population. We examined the relation between both behavioral and questionnaire measures of effortful control and densely-sampled, stressinduced cortisol trajectories in American and Chinese preschoolers.

- The findings from this study are the first to suggest that Western and non-Western preschool children have different predictors of their stress-related cortisol response.

- Emerging emotion regulation systems appear to be impacted by culture both very early in childhood and at a physiological level.

Culture clearly influences human development, but does it affect physiological responses to stressful events? Our main goal was to test the cross-cultural generalizability of a salient model of early self-regulation: how effortful control (EC) skills relate to young children's cortisol secretion following negative emotion induction. Children who fail to develop the skills to adequately regulate their behavior and emotions are at risk for a host of later negative developmental outcomes including poor peer adjustment (Kochanska, Murray, \& Harlan 2000), low levels of prosocial behavior (Kochanksa, Murray, Jacques, \& Vandegeest, 1996), and myriad behavior problems (Olson et al., 2005). Clearly, understanding the processes underlying children's ability to regulate negative affect is an issue of great theoretical and practical importance. However, relatively little research has been devoted to understanding cultural differences in the basic processes underlying children's ability to regulate negative experiences. An objective of the current study was to build on previous research on effortful control differences in U.S. and Chinese children and to examine, more carefully, how EC relates to biological underpinnings of emotion regulation (ER) across two distinctive cultural groups.

In order to examine how complex models of stressregulation might be moderated by culture, we first examine the literature on emotion regulation and effortful control from a general perspective. We begin by

Address for correspondence: Adam S. Grabell, University of Michigan, Ann Arbor, Michigan, USA; e-mail: grabell@umich.edu 
briefly discussing what is known about emotion regulation and its relation to the hypothalamic-pituitaryadrenal (HPA) axis. Next, we describe and delineate multiple subcomponents of effortful control and how these skills may relate to HPA axis activity during an emotional challenge. Finally, we examine the role of culture by considering how early socialization experiences in Western and non-Western countries might impact effortful control, emotion regulation, and their interaction.

\section{Emotion Regulation and the HPA Axis}

The construct of emotion regulation encompasses processes necessary for voluntarily modulating the intensity, time-course, and valence of emotional experiences (Thompson, 1994). Competence in managing negative emotions, such as feelings of disappointment and anger, is a crucial milestone of early childhood (Calkins \& Hill, 2007; Kopp, 1989). Furthermore, emotion regulation is goal oriented. Children and adults are motivated to manage negative emotions, either automatically or effortfully, to achieve an adaptive outcome (Gross, 1998; Izard, 2009). For example, suppose a preschool teacher forgets to give a child a turn in a group activity. In the child's attempt to rectify the situation, one would expect employment of strategies to move into a less distressing emotional state, and/or demonstrate behavioral competence matching the demands of the situation. In the present study, preschool children in the U.S. and China were exposed to an emotional challenge designed to elicit feelings of disappointment and frustration, not unlike those experienced in a typical preschool environment, in the presence of an unfamiliar adult.

Emotion regulation plays out dynamically across multiple systems, including neurobiological processes (Thompson, 2011) that all humans possess. For instance, the activity of the hypothalamic-pituitary-adrenal (HPA) axis is central to the neurobiology of emotion regulation. When humans experience psychological stress, including onset of negative emotion, a cascade of signals from the hypothalamus to the adrenal glad, via the pituitary gland, results in the release of the glucocorticoid cortisol (Gunnar \& Vasquez, 2006). Cortisol levels typically rise and then return to baseline levels in the minutes following a psychosocial stressor (Dickerson \& Kemeny, 2004). Furthermore, individuals experiencing high levels of psychosocial stress have shown intensified cortisol reactivity and a longer recovery process than others (Earle, Linden \& Weinberg, 1999; Dickerson \& Kemeny, 2004). Therefore, in the present study we conceptualize children's cortisol reactivity and recovery following an emotional challenge as a biological marker of emotion regulation.

The prefrontal cortex (PFC) has been hypothesized to play a critical role in this process. For example, functional differences in the PFC have been linked to individual differences in the rate of cortisol production and return to baseline levels of output (Sullivan et al., 2004; Ulrich-Lai \& Herman, 2009). Moreover, the PFC has anatomical connections to both the amygdala, the primary area of the brain involved in emotional responding, as well as the HPA axis via the hypothalamus (Sullivan, 2004), and appears to be an important way station between neural and neuroendocrine aspects of emotional responses.

Individual differences in HPA axis regulation are evident in infancy (Gunnar \& Donzella, 2002; Luijk et al., 2010) and parental responses interact with children's physiology to further affect regulatory responses (Essex et al., 2011). However, the cognitive processes and temperamental characteristics that underlie children's ability to modulate their physiological response to negative emotion remain largely unexplored, particularly cross-culturally.

\section{Effortful Control}

Individual differences in children's emotion-related cortisol responses may be related to the temperament construct of effortful control (Rothbart \& Rueda, 2005). Rothbart and colleagues (2000) have defined effortful control as the composite of two separate but related skills: inhibitory control (IC), the capacity to plan and suppress approach responses under instruction or in novel or uncertain situations; and attentional focusing (AF), the capacity to maintain attentional focus on taskrelated stimuli. Inhibitory control and attentional focusing are also considered to be fundamental processes in what has generally been termed executive functioning (EF), which also includes working memory capacity (Zelazo, Müller, Frye, \& Marcovitch, 2003; Best \& Miller, 2010). Effortful control variables in the present study can thus be argued to fit into more than one theoretical construct. In the literature review that follows, we discuss studies relevant to the constructs of $\mathrm{EC}$ and EF with a particular focus on IC and AF, components of both constructs.

In the present study we propose, as others have (see Thompson, 2011), that attentional and inhibitory skills are embedded within children's emotion regulation strategies. Indeed, the PFC plays an important role in both emotion regulation and cognitive control skills such as inhibition of inappropriate responses (Garavan, Ross, 
\& Stein, 1999). Therefore, we expected that individual differences in effortful control would be associated with differences in the rate of cortisol production following an emotional challenge. Blair, Granger, and Razza (2005) found that U.S. preschoolers who performed more poorly on attention-shifting and inhibitory control tasks had higher cortisol levels during the testing session. However, Blair et al.'s study examined cortisol production under conditions that were cognitively, but not necessarily affectively, challenging. Thus, the relevance of cortisol secretion to emotion regulation and effortful control under affectively or motivationally laden conditions was unclear. Nonetheless, the results from Blair et al.'s study suggest that there are clear relations between cortisol output and components of EC.

Moreover, studies in U.S. preschoolers have shown that lower levels of parent-rated EC are associated with higher cortisol levels during peer interaction (Gunnar, Sebanc, Tout, et al., 2003), following a frustrating event (Spinrad et al., 2009), and diurnally (Dettling, Gunnar, \& Donzella, 1999). Thus, children with poorer cognitive regulatory skills may demonstrate less competent ER and higher physiological reactivity to emotional stress than others, in part due to these shared neural networks (Posner \& Rothbart, 2000). However, this hypothesis has been incompletely tested and, more specifically, the subcomponents of EC that might relate to physiological subcomponents of ER have not been clearly delineated in either Western or non-Western samples.

As stated previously, EC is a heterogeneous construct, comprising inhibitory control and attentional focusing. Furthermore, in some measures, such as the Child Behavior Questionnaire (CBQ), effortful control is rated in motivationally-laden situations per parent report across a broad set of contexts (Rothbart, Ahadi, Hershey, \& Fisher, 2000). In other studies, these skills are assessed during performance-based tasks in a much more narrow context (Kim et al., 2013). A lack of consistency in how EC is operationalized across studies is potentially problematic for developmental research in general, but particularly so for cross-cultural research. Thus, we chose to directly test children's inhibitory control abilities across a set of three tasks used in studies with both U.S. and Chinese children (e.g., Carlson \& Moses, 2001; Sabbagh, Xu, Carlson, Moses, \& Lee, 2006; Tardif, So, \& Kaciroti, 2007), which we used to form a composite score. Moreover, previous researchers have used the CBQ as a parent-report measure of effortful control with both U.S. and Chinese children (Ahadi, Rothbart, \& Ye, 1993; Eisenberg et al., 2007; Zhou, Lengua, \& Wang, 2009). Therefore, we were able to utilize a variety of existing measures and tasks known to be reliable across Western and non-Western samples.
Finally, within and across cultures, there remain notable gaps in our understanding of how effortful control and children's emotion-related cortisol response may be connected. To our knowledge, no previous investigators have examined relations between parent report and behavioral measures of effortful control and children's cortisol responses elicited during an emotionally challenging task. In addition, the relation between effortful control and emotion-related cortisol has yet to be tested in process models that examine physiological regulation in terms of both its rise and recovery phases. Thus, it is unknown whether effortful control subcomponents predict the rate of both rise and fall in children's cortisol responses after an emotional challenge. As mentioned above, because EC is a heterogeneous construct comprising different abilities, studies are needed to test whether the subcomponents of effortful control: inhibitory control and attentional focusing, relate differently to aspects of the cortisol response. Finally, as shown below, there is little understanding of the cross-cultural generalizability of our research models, particularly how effortful control subcomponents relate to physiological measures of emotion regulation across cultures.

\section{Assessing Emotion Regulation and Effortful Control Processes Across Different Cultural Contexts}

Despite the dramatic rise in research on emotion regulation (Gross, 2009), most studies continue to focus on children growing up in Western European cultures. Given that early emotion regulation skills underpin children's social, emotional, and academic development, it is important to examine ER in young children growing up in diverse populations, including non-Western countries. In particular, there are characteristics of East Asian cultures, including the People's Republic of China, which may have profound effects on the socialization of emotion regulation (Tardif, Wang, \& Olson, 2009). As is the case in all cultures, Chinese children's emerging emotion regulation skills are intentionally shaped and scaffolded through interpersonal relationships with caregivers and authority figures (Eisenberg, Chang, Ma, \& Huang, 2009). In China, these processes are hypothesized to occur in a more collectivistic-valued framework when compared to Western cultures (Chao, 1994).

Consistent with this orientation, Chinese parents more rigorously endorse values and parenting strategies that foster cooperation, suppress anger and aggression, and encourage impulse control in their children (Chao et al., 2000; Liu et al., 2005; Wang, Chen, Chen, Cui \& Li, 2006). Chinese mothers, when compared to 
European-American mothers, are more likely to attribute their preschoolers' dysregulated emotions, such as acts of aggression, to externally motivated causes and assume that they are malleable rather than dispositional (Cheah \& Rubin, 2004). These values and caregiving goals extend to teachers and the school environment in China (Phelps, 2005), and have been described as products of a broader societal emphasis upon prohibiting disruptive behaviors and maintaining social harmony (Chen, $\mathrm{Cen}, \mathrm{Li}, \& \mathrm{He}$, 2005). In contrast, European-American mothers tend to be more accepting of rough and tumble play, noncompliance, and other harmony-disrupting behaviors in the interest of encouraging children to express their individual feelings in social situations (Jose, Huntsinger, Huntsinger, \& Liaw, 2000; Liu et al., 2005; Suizzo net al., 2008).

Given cultural differences in how early emotion regulation skills are valued and reinforced, we would expect that Chinese and U.S. preschoolers might react differently, at least in terms of their outward emotional expression and behavior, to an interpersonal stressor involving an unfamiliar adult. However, this does not mean we can assume a direct translation to cross-cultural differences in physiological indicators of emotion regulation such as cortisol reactivity and/or recovery. Similarly, it is not clear whether subcomponents of effortful control relate to physiological markers of ER similarly across cultures.

Because of the strong emphasis on self-control in Chinese child-rearing practices (Cheah \& Rubin, 2003; $\mathrm{Xu}$ et al., 2005), one would expect that preschool children in China would perform better, and be rated higher, on EF and EC skills than their U.S. counterparts. Indeed, Chinese preschoolers perform better than U.S. preschoolers on EF batteries measuring inhibition and attention (Sabbagh, et al., 2006; Lan, Legare, Ponitz, Li, \& Morrison, 2011). However, cross-cultural studies looking at parent and teacher ratings of inhibitory control and attentional focusing have been mixed, with some studies showing that Chinese parents rate their children higher on these scales (Ahadi, Rothbart, and Ye, 1993) and other studies showing no differences (Zhou et al., 2009). Thus, cross-cultural differences in effortful control may depend on the method of assessment and the aspects of these skills examined. In particular, Sabbagh et al. (2006) used Stroop-like behavioral measures of inhibitory control, whereas Rothbart and Zhou used parental reports of children's inhibitory and attentional control in a broader set of contexts. A strength of the current study, therefore, is the use of multiple measures of EC in preschool-aged children across the two cultures.

Importantly, cross-cultural research on how effortful control subcomponents relate to other measures of wellbeing, such as academic performance and behavior problems, has only just begun. In the above-mentioned study by Lan et al. (2011), although Chinese preschool children performed better than U.S. children on inhibition, working memory, and attentional control tasks, performance on these tasks was associated with academic achievement in both cultures. In the Sabbagh et al. (2006) study, moreover, both groups had a similar correlation between executive function performance and Theory of Mind (ToM) ability (but see Tardif et al., 2007, for the lack of a correlation between EF and ToM in Hong Kong Chinese preschoolers). Similarly Olson and colleagues (2011) found that a composite of preschooler's CBQ inhibitory control and attentional focusing subscale scores, along with their performance on three Stroop-like tasks, was inversely related to high levels of externalizing problems (maternal ratings) in U.S. and Chinese preschool children. However, Ahadi and colleagues (1993), using just the CBQ subscales, found differences in how these constructs were related to children's behavior problems across cultures. Specifically, U.S. children with higher ratings of effortful control expressed lower levels of negative affect, but this same relation did not hold true for China.

Thus, individual differences in the various subcomponents of EC might show similar or different associations with physiological markers of ER in different cultures. Furthermore, models of emotion regulation may appear similar or different across cultures depending on whether they are examined using parent report, behavioral, or physiological levels of analysis. Ideally, and in order to distinguish differences in the relations across subcomponents of EC and ER, all three levels of analysis should be examined (Beauchaine, 2009). However, to date this has not been done in either U.S. or cross-national studies of preschoolers' effortful control and emotion regulation.

\section{Goals of the Current Study}

Our main objective was to test whether superior effortful control abilities predicted a more modulated cortisol trajectory following an emotional challenge in both U.S. and Chinese preschool children. Specifically, we examined whether higher levels of inhibitory control and attentional focusing predicted lower levels of physiological regulation to a stressful event, marked by: 1) a shallower, more gradual rise in cortisol and 2) a steeper, more dramatic return to baseline cortisol levels, in both cultures, after controlling for non-verbal IQ, age, and the time elapsed between waking and testing. Our model reflects a hypothesized underlying biological mechanism which would generally predict that EC skills would relate to ER similarly in both Western and non-Western children. However, it is also possible, as we have seen 
in other studies examining only a subset of these measures, that these constructs may relate to each other differently across the two cultural groups.

We addressed these gaps in knowledge by administering identical multi-measure assessments of effortful control and emotion regulation to preschoolers growing up in the U.S. and China. Both behavioral and parent report measures were used to assess preschoolers' effortful control. To examine individual differences in children's emotion-related cortisol response, we administered an emotionally challenging task and sampled salivary cortisol at baseline and every ten minutes afterwards. Cortisol was densely sampled to assess physiological activity associated with children's emotional reactivity to and recovery from the event. Dense and prolonged cortisol sampling, as opposed to collecting cortisol at only one time point, provides data that are optimal for understanding complex processes of reactivity and recovery following stressful activation (LopezDuran et al., 2009). To our knowledge, our study is the first to examine the relation of individual differences in inhibitory control, attentional focusing, and stress reactivity and recovery in both Western and non-Western preschool children, and is the first to test this relation using both maternal ratings and performance based scores of EC in Chinese and U.S. preschoolers.

Finally, an additional goal was to examine how the subcomponents of effortful control, inhibitory control and attentional focusing, related to each other and to emotion-related cortisol across cultures in both parentrated and performance-based contexts.

\section{Method}

\section{Participants}

Participants were 117 preschool-age children from the U.S. and China. Demographic data, organized by country, are shown in Table 1. U.S. participants included 59 preschoolers from a small Midwestern city (28 males). U.S. participants were recruited from 15 preschools within a 30-minute driving radius through preschool visits, fliers, and newspaper ads. The average child age was 53 months ( $S D=4$ months) and age ranged from 44 to 66 months, although the 66-month-old was later dropped from the study for being out of age range. With the 66-month old excluded, the age range was 44 to 63 months. Furthermore, one child in the U.S. sample failed to provide any cortisol data and therefore 57 children were examined the present study. The sample was $72.4 \%$ Caucasian, 10.3\% African American, 8.6\% Mixed Ethnicity, 3.4\% Other Ethnicity, 1.7\% Hispanic, and 3.4\%
Table 1 Demographic Data: Study Participants by Culture

\begin{tabular}{|c|c|c|c|c|c|}
\hline \multirow[b]{2}{*}{ Variables } & \multicolumn{2}{|c|}{$\begin{array}{l}\text { United States } \\
\quad(N=57)\end{array}$} & \multicolumn{2}{|c|}{ China $(N=60)$} & \multirow[b]{2}{*}{$\mathrm{p}$} \\
\hline & $M$ & $S D$ & $M$ & $S D$ & \\
\hline Age (months) & 53.7 & 4.79 & 52.5 & 3.38 & ns \\
\hline Gender ( $\%$ male $)$ & $49 \%$ & & $48 \%$ & & ns \\
\hline Age Mother & 36.36 & .67 & 33.14 & .37 & $\mathrm{p}<.001$ \\
\hline Age Father & 37.45 & .97 & 35.8 & .45 & $\mathrm{p}<.05$ \\
\hline \multicolumn{6}{|l|}{ Socioeconomic Status } \\
\hline Mother education & 6.23 & .84 & 5.42 & 1.12 & $\mathrm{p}<.001$ \\
\hline Father education & 6.02 & 1.1 & 5.64 & 1.15 & \\
\hline $\begin{array}{l}\text { Mother full-time } \\
\text { employment }(\%)\end{array}$ & $60.3 \%$ & & $76.7 \%$ & & $\mathrm{p}<.001$ \\
\hline $\begin{array}{l}\text { Father full-time } \\
\text { employment }(\%)\end{array}$ & $70.7 \%$ & & $88.3 \%$ & & ns \\
\hline Number of siblings & 1.11 & .725 & .04 & .189 & $\mathrm{p}<.001$ \\
\hline Married (\%) & $75.9 \%$ & & $91.7 \%$ & & $\mathrm{p}<.01$ \\
\hline
\end{tabular}

Unreported. On average, U.S. children had one additional sibling. Among U.S. mothers, 75.9\% reported living in a dual parent household, $12.1 \%$ reported they were divorced, $1.7 \%$ reported they were single, and $10.3 \%$ did not respond. In the U.S. sample, deliberate efforts were made to recruit families from lower socioeconomic backgrounds in order to have a more diverse and representative sample. In addition, 14 children were enrolled in regular half-day (approximately three hours per day) preschool, while the rest of the U.S. children were enrolled in preschool full-time. Because the amount of time a child spends in a preschool environment may affect socialization, self-regulation skills, and daytime cortisol levels (Dettling et al., 1999), we compared half-time and full-time U.S. preschool children on all variables used in the present analyses. There were no significant differences between half-time and full-time U.S. preschoolers on any of the study variables, including their cortisol levels following the emotional challenge.

In Beijing, China, 60 children (29 males) were recruited from three preschools in the Southern and Western districts of Beijing. The mean age was 52 months ( $S D=3$ months) and age ranged from 47 to 61 months. Because of China's one-child policy, only two of the children (a pair of twins) were reported to have siblings. All families were Han Chinese. In addition, $91.7 \%$ of Chinese mothers reported living in a dual parent household, while $8.3 \%$ did not report their marital or living status. All Chinese mothers reported enrolling their child in full-time preschool.

As shown in Table 1, mothers and fathers in the U.S. were slightly, but significantly, older than their Chinese counterparts. Mothers in the U.S. reported more formal schooling than Chinese mothers, largely due to a higher percentage of American mothers achieving graduate school-level degrees. In both samples, over $50 \%$ of 
mothers reported completing at least two to three years of college. Chinese mothers were more likely to report being employed full-time than U.S. mothers, but in both samples the majority reported full-time employment. There were no differences in education or full-time employment between U.S. and Chinese fathers. Moreover, although the Chinese children were from a major urban center, the children were sampled from schools that were affiliated with local universities and located in relatively more affluent and suburban areas of the city rather than the center of the city. In this way they were more comparable to preschoolers in our Midwestern university town than would otherwise be suggested by this sampling difference.

\section{Procedures}

As part of a larger study, all children were tested in their preschool setting across a period of three consecutive days. Testing sessions were approximately two hours long. The effortful control tasks were administered to children on all three days, one task per day. The emotion regulation task was administered on the second or third day (counter-balanced) with cortisol sampled densely during the testing session. Mothers completed questionnaires on family demographics and child behavior.

\section{Emotion regulation task}

All children completed a disappointing prize task where they ranked a group of prizes from most preferred to least preferred with a familiar experimenter (Cole, 1986; Saarni, 1984). "Good" prizes were toys such as model fire trucks and bubble kits while "bad" prizes were objects such as broken combs and baby toys. The familiar experimenter left and, a short while later, an unfamiliar experimenter entered the room, told the child they had won their highest ranked prize, but proceeded to hand the child the lowest ranked prize instead. The unfamiliar experimenter acted unaware that they had committed any error and ignored the child for 60 seconds. The unfamiliar experimenter then left the room, without interacting with the child, and the child was left alone for an additional 60 seconds. The disappointment was resolved when the familiar experimenter returned, asked if the child was happy with his/her prize, brought in the unfamiliar experimenter, gave the child the option of taking the highest ranked prize, and apologized for the confusion. The entire task lasted 5 to 8 minutes. After completing the task, children spent 30 minutes watching soothing, calming, age-appropriate videos followed by the performance-based EC tasks and other measures.

\section{Cortisol sampling}

Salivary cortisol was collected 30 minutes prior to the beginning of the disappointment task, and then every 10 minutes from the onset of the disappointing event until 90 minutes post-disappointment. The purpose of taking a sample 30 minutes prior to the onset of the disappointment paradigm was to observe cortisol levels and to provide an opportunity for them return to baseline after potentially arousing activities that took place in the children's preschool settings prior to the study, as well as any anticipatory anxiety or excitement the children might have had about the study session. To avoid chemical interference, no stimulant was used to generate saliva. Research assistants chewed dental rolls in parallel with the children for 60 seconds and used sticker boards as motivation to ensure compliance in collecting the samples. The cotton dental rolls were then inserted into a plastic salivette and refrigerated until centrifuged. Each sample was centrifuged for five minutes within 24 hours of collection and assayed in duplicate using commercial kits (High Sensitivity Salivary Cortisol Enzyme Immunoassay Kit, Salimetrics). The same make of salivettes and model of assay kit was used at both sites. Time elapsed between morning waking and collection of cortisol was recorded and entered as a covariate to control for effects of diurnal cortisol on the stress response.

\section{Effortful control behavioral tasks}

We used three tasks to assess behavioral measures of inhibitory control: the "Grass/Snow Task," "Luria's Hand Game," and "The Day/Night Stroop Task." Each task involved an initial imitative phase, where the child copied or followed the experimenter, followed by a conflict phase, where the child did the opposite of the experimenter. These three tasks were presented to all children in a fixed order (Day One: Grass/Snow, Day Two: Luria's Hand Game, Day Three: Day/Night Stroop).

During the Grass/Snow task (Carlson \& Moses, 2001), the experimenter showed the child a board with a white square and a green square, and made sure the child knew the color of grass and snow. During the imitative phase, the child was instructed to point to the white square when the experimenter said "snow" and to the green square when the experimenter said "grass," for 10 trials. During the conflict phase, the child was instructed to point with the opposite kind of point for 10 trials.

During Luria's Hand Game (Luria, Pribram, \& Homskaya, 1964), the experimenter pointed with either one or two fingers. During the imitative phase, the child was instructed to make the same kind of point for 10 
trials. During the conflict phase, the child was instructed to make the opposite kind of point for 10 trials.

During the Day/Night stroop task (Diamond \& Taylor, 1996), the experimenter had a picture book of suns and moons. During the imitative phase, the child was supposed to say "day" when the experimenter revealed a sun picture, and "night" when the experimenter revealed a moon picture for 10 trials. During the conflict phase, the child was instructed to say "night" when the experimenter revealed a daytime scene, and "day" when the experimenter revealed a nighttime scene for 10 trials.

All three tasks were videotaped and scored manually both at the time of administration and using the video footage. Any discrepancies between the two sets of scores were resolved using the video footage. Because there was no meaningful variation for the imitative phase of the three tasks for either culture due to ceiling effects, z-scores were calculated from the number of errors in the conflict phase only, for each task, and then summed across tasks to create a behavioral inhibitory control composite score.

\section{Parent Questionnaires}

\section{Component effortful control skills}

Mothers completed the Child Behavior Questionnaire (Rothbart, 1989), a 109-item measure of child temperament. Parents were asked to rate how much each question applied to their child on a 7- point scale. The Inhibitory Control and Attentional Focusing subscales of the CBQ are the two subcomponents of effortful control (Rothbart, Ahadi, Hershey, and Fisher, 2003). In the current study, the CBQ was translated and back-translated by Mandarin-Cantonese and Mandarin-English bilinguals at both the U.S. and Beijing sites; discrepancies were discussed and resolved between translators.

\section{Inhibitory Control}

Inhibitory Control is a rating of the child's capacity to plan and suppress inappropriate actions (e.g. "Can lower his/her voice when asked to do so," "Can wait to enter new activities if s/she asked to"). Inhibitory Control had good reliability in the U.S. sample and moderate reliability in the Chinese sample $(\alpha=.77, .62$, respectively). Although the reliability was lower for the Chinese sample, it is important to note that other studies with older Chinese children have reported reliabilities for the CBQ IC scale in the same range (e.g., .61 in Zhou et al.'s (2009) sample of 382 school-aged Chinese children and
.71 in Eisenberg et al.'s (2007) study of 697 Chinese first and second graders).

\section{Attentional Focusing}

Attentional Focusing indexes the child's distractibility vs. capacity to become engaged or engrossed in a task (e.g. "Has trouble concentrating when listening to a story" [Reversed], "Sometimes becomes absorbed in a picture book and looks at it for a long time"). Reliability for Attentional Focusing was good in both the U.S. and Chinese samples ( $\alpha=.80, .84$, respectively). Because the IC and AF scales tap two subtly different skills (inhibition versus sustained attention), they were examined separately in analyses.

\section{Covariates}

Children completed the Block Design subtests from the Wechsler Preschool and Primary Scale of Intelligence-III (WPPSI-III) (Wechsler, 2002). Because norms do not exist for Chinese children, we used raw Block Design scores and entered age as an additional covariate for both cultures.

The stress-related cortisol response is influenced by an individual's diurnal cortisol cycle (Buckley \& Schatzberg, 2005; Schmidt-Reinwald et al., 1999). Therefore, we controlled for the time elapsed between waking and cortisol sampling.

\section{Preliminary Analyses}

After assay, cortisol values at each time point was entered into an Excel spreadsheet and an automated algorithm was used to identify the following key points in the cortisol secretion curve: the first peak value, pre-peak nadir, and post-peak nadir (see Figure 1). Specifically, peaks and nadirs were defined as points that were 5\% greater in magnitude (either positively or negatively) than neighboring points. A "reactivity" slope was defined as the change in cortisol elevation from the pre-peak nadir to the first peak divided by the time between these two events. A "recovery" slope was similarly defined as the change in cortisol elevation from the first peak to postpeak nadir divided by the time between these two events.

\section{Missing cortisol data and normality}

The fraction of missing data for the cortisol responses was small. Six U.S. children had missing cortisol data for three or fewer points. In the Chinese sample, one child 


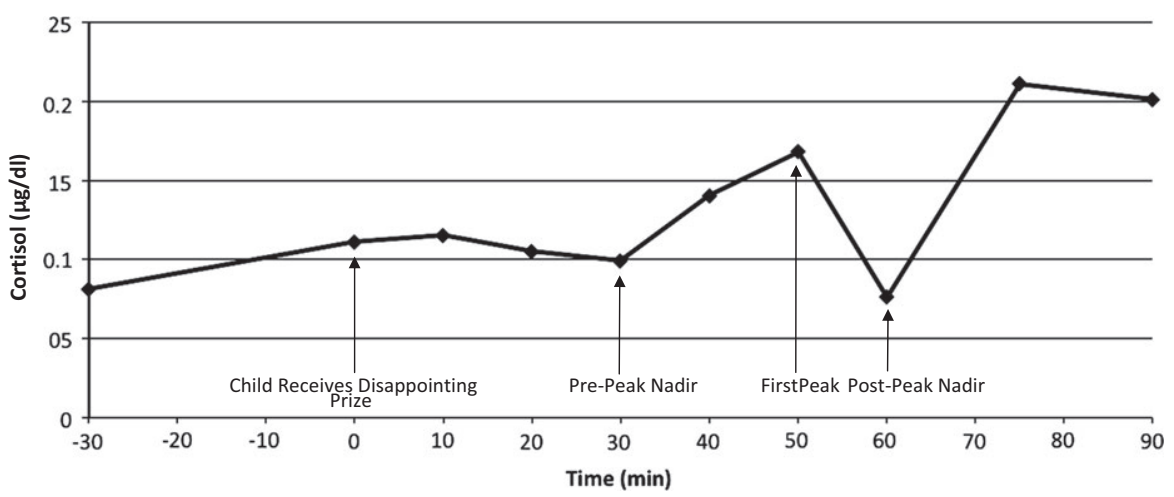

Figure 1 Raw cortisol elevations (micrograms per deciliter) at each time point for a single participant completing the Prize Task. Arrows indicate key events in the task and points used to create slope values.

Note. Many children produced an increase in cortisol after the post-peak nadir. This activity is related to the child returning to the classroom to complete additional cognitive and behavioral tasks.

had missing cortisol data for six of the collection points. Missing cortisol data were imputed using the IVEWARE (Raghunathan et al., 2002) SAS macro following a multivariate regression model with the missing values imputed sequentially based on the observed values from both within and across the tasks. Data were log transformed for the imputation process to improve the normality and transformed back to the original scale after the imputation. Imputation was performed separately for each culture. Consistent with previous research (Gunnar \& Vazquez, 2001), distribution of the dependent cortisol variables was skewed. A $\log 10$ transformation was used to successfully bring cortisol variable distributions within normal limits.

\section{Analysis}

Structural Equation Modeling (SEM) using MPlus (Muthén \& Muthén, 1998) was used to test the relation between EC subcomponents and emotion-related cortisol slopes. A major advantage of using SEM as opposed to multiple regression is the ability to apply the model to multiple groups simultaneously and observe how the model fit changes when path parameters are constrained to be equal or allowed to be free. Missing data were handled using Full Information Maximum Likelihood (FIML). First, in order to examine how EC subcomponents and cortisol variables related to each other in each culture, we fit the covariance matrices of the two groups simultaneously to the same model while allowing all paths and covariances to be free, producing a fully saturated model. Next, to test whether our model generalized across cultures, we constrained all direct paths and covariances to be equal between the two groups and examined change in model fit using the change in Chi-square test. Finally, to determine which parts of our model were different between cultures, we constrained and freed each path in sequence to determine which parameters were significantly different between the U.S. and Chinese samples. Individual change in Chi-square tests were used to determine which specific parameters of the model showed the most robust group differences.

\section{Results}

Table 2 shows descriptive data for the study variables in the U.S. and Chinese samples. U.S. and Chinese children did not differ in their age, gender ratio, or cortisol

Table 2 Comparison of study variables between US and Chinese samples

\begin{tabular}{|c|c|c|c|c|}
\hline & $\begin{array}{l}\text { United States } \\
\begin{array}{c}(N=57) \\
M(S D)\end{array}\end{array}$ & $\begin{array}{c}\text { China }(N=59) \\
M(S D)\end{array}$ & $p$ & $d$ \\
\hline $\begin{array}{l}\text { CBQ-Inhibitory } \\
\text { Control }\end{array}$ & $4.85(.78)$ & $4.61(.65)$ & $<.08$ & .33 \\
\hline $\begin{array}{l}\text { CBQ-Attentional } \\
\text { Focusing }\end{array}$ & $4.83(.768)$ & $4.60(.760)$ & ns & \\
\hline $\begin{array}{l}\text { Lab based } \\
\text { Inhibition }\end{array}$ & $21.94(7.45)$ & $24.67(4.2)$ & $<.05$ & .45 \\
\hline $\begin{array}{l}\text { Cortisol reactivity } \\
\text { slope }\end{array}$ & $.0019(.002)$ & $.003(.004)$ & ns & \\
\hline $\begin{array}{l}\text { Cortisol recovery } \\
\text { slope }\end{array}$ & $-.002(.002)$ & $-.002(.002)$ & ns & \\
\hline $\begin{array}{l}\text { Time between } \\
\text { waking and } \\
\text { testing (minutes) }\end{array}$ & 278 (191.9) & $289.3(195.03)$ & ns & \\
\hline $\begin{array}{l}\text { Block design raw } \\
\text { score }\end{array}$ & $15.17(6.77)$ & $19.03(5.44)$ & $<.01$ & .63 \\
\hline
\end{tabular}

Note Z-scores were used in regression models for independent and control variables, unstandardized scores are presented here for interpretation; $\mathrm{a}=\mathrm{T}$-score of $44, \mathrm{~b}=\mathrm{T}$-score of $51, \mathrm{c}=\mathrm{T}$-score of $47, \mathrm{~d}=\mathrm{T}$ score of 56 
reactivity or recovery values. U.S. preschoolers had significantly lower non-verbal IQ, and more errors on the inhibitory control behavioral tasks, but higher ratings on the CBQ-Inhibitory Control subscale compared to Chinese preschoolers.

\section{Manipulation Check and Overt Facial Expressions}

The entire prize task was videotaped in both cultures. Coders at each site scored positive and negative affect during the task from video footage. Each coder coded at least five children from the other culture for reliability purposes. Reliability (inter-coder correlation) was acceptable for both groups (Positive affect: US $=.69$, China $=.79$; Negative affect: US $=.68$, China $=.76$ ). In findings previously reported elsewhere (Miller et al., 2012) both U.S. and Chinese preschoolers showed an increase in negative affect after the onset of the disappointment. Furthermore, Chinese preschoolers showed significantly less positive and negative affect, and more "neutral" facial expressions, than U.S. preschoolers following the onset of the disappointing event.

\section{Gender as a Potential Moderating Effect}

In both cultures, we tested whether interaction terms for gender and CBQ-IC and AF, as well as the more cognitive inhibitory control behavioral composite, correlated with cortisol reactivity or recovery. Gender and each of the EC subcomponent scores were centered and multiplied together to create interaction terms. No significant gender effects were found with cortisol. Thus, interaction terms were not included in the path model.

\section{Additive Model Predicting Emotion Regulation- Related Cortisol Patterns}

As shown in Figure 2, when the entire model was applied to each group separately, CBQ-IC significantly predicted lower cortisol reactivity in the U.S. sample $(\beta=-.308, p<$ .05 ) but not in the Chinese sample. Conversely, CBQ-AF significantly predicted greater cortisol reactivity in the Chinese sample $(\beta=.424, p<.01)$, but not the U.S. sample. Thus, greater CBQ-IC predicted more shallow reactivity for U.S. children, whereas, surprisingly, greater CBQ-AF predicted steeper reactivity for Chinese children. In addition, CBQ-IC predicted steeper cortisol recovery for the U.S. sample $(\beta=-.306, \mathrm{p}<.05)$, but showed no association with cortisol recovery in the
Chinese sample. These data suggest that U.S. children who were rated higher on CBQ-IC returned to baseline cortisol levels more quickly. Performance-based inhibitory control did not predict cortisol reactivity or recovery for either culture. The amount of cortisol reactivity variance accounted for in the model was marginal for both cultures (US: $\mathrm{R}^{2}=.163$; China: $\mathrm{R}^{2}=.159, \mathrm{p}<.10$ ). Moreover, cortisol recovery variance accounted for was marginally significant only for the U.S. sample $\left(\mathrm{R}^{2}=\right.$ $.151, \mathrm{p}<.10$ ).

When the entire model was constrained to be equal between groups, the model fit became significantly worse $\left(\Delta \chi^{2}=64.65, \mathrm{df}=37, \mathrm{p}<.01\right)$, suggesting that the best model is one in which parameters are estimated separately by culture.

Finally, in order to determine whether individual path coefficients differed by culture, and how individual paths contributed to the overall poor fit of the fully constrained model, we constrained and unconstrained each path sequentially. As shown in Figure 2, change in Chisquare tests revealed that the direct path from CBQ-AF to cortisol reactivity was marginally significantly different between the two cultures $\left(\Delta \chi^{2}=3.52, \mathrm{df}=1, \mathrm{p}=.06\right)$. Additionally, marginal differences were found between cultures in the correlation coefficients for CBQ-AF with the inhibitory control behavioral task composite $\left(\Delta \chi^{2}=\right.$ 3.51 , df $=1, \mathrm{p}=.06$ ), and the inhibitory control behavioral task composite with non-verbal intelligence $\left(\Delta \chi^{2}=3.81\right.$, df $\left.=1, \mathrm{p}=.05\right)$, such that these relations were stronger for U.S. children. Finally, we found a marginal difference between cultures in the correlation coefficient for age and time awake $\left(\Delta \chi^{2}=3.34, \mathrm{df}=1, \mathrm{p}=\right.$ .06 ), which had a stronger association for Chinese children. Although change in Chi-square tests for these paths were not significant at the $p<.05$ level, they indicate which paths from the completely constrained model were an especially poor fit.

\section{Post-hoc analyses}

Opposite patterns of association between children's EC skills and physiological reactivity to stress may reflect cultural differences in coping strategies. Studies on Western samples have shown that children with higher levels of internalizing problems, such as anxiety, exhibit both an attentional bias towards negative information (Martin, Horder, \& Jones, 1992; Vasey, Daleiden, Williams, \& Brown, 1995), and a stronger physiological response to upsetting events (Kagan et al., 1987). Although Chinese mothers did not rate their children high on internalizing problems on a translated version of the Child Behavior Checklist, Preschool Version (Achenbach \& Rescorla, 2000), the opposite patterns of 


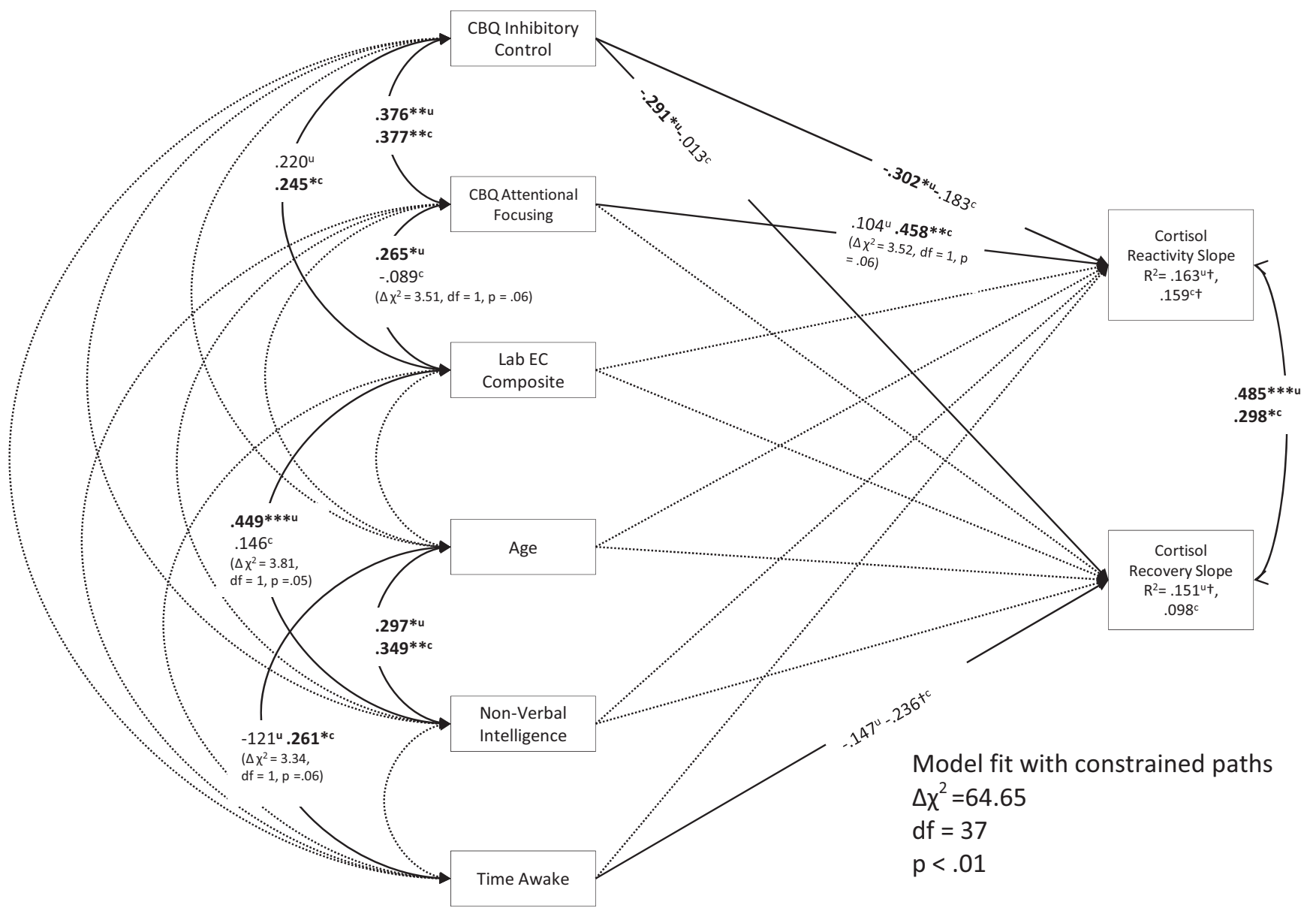

$+p<.10 ; p<.05 ; * * p<.01 ; * * p<.001$

Figure 2 Path model parameter estimates by culture (United States $N=57$; China $N=60$ ).

Note. u,c denote US and China sample parameter estimates, respectively, for the unconstrained model. Dotted paths denote nonsignificant pathways for both groups, while solid-line paths denote significant pathways for one or both groups. Significantly different path coefficients are denoted in parentheses.

association across cultures could potentially reflect cultural differences in an emphasis on suppressing the expression of negative emotions. Thus, we controlled for CBCL internalizing problem scale scores in the model as a post-hoc test. However, even after controlling for maternal-rated internalizing problems, results showed that the relation between attentional focusing and cortisol reactivity remained significant at the $\mathrm{p}<.05$ level for the Chinese preschoolers.

Because our pattern of findings could have been impacted by a subset of children with unusually high or low cortisol reactivity, we tested whether different levels of cortisol activation drove the differential relation between AF and cortisol reactivity across the two groups of preschoolers. We performed a median split on the
Chinese sample based on their cortisol reactivity values, creating "high" and "low" reactivity groups. A one-way ANOVA revealed that the high and low groups were significantly different in their cortisol reactivity values $(F$ $=84.02, \mathrm{p}<.001)$, but were not different in their CBQ-IC or AF scores, their scores on the lab-based tasks, age, non-verbal intelligence, or time between waking and testing. Furthermore, the bivariate correlation between $\mathrm{AF}$ and cortisol reactivity was essentially the same in both groups (Low: $r=.344$; High: $r=.322$ ), but did not reach significance in either group due to the small sample size in each subgroup. A similar comparison was conducted, regardless of culture, for the children who had relatively higher versus relatively lower cortisol reactivity, and obtained similar results. 


\section{Discussion}

Our main goal was to test the generalizability of a complex model of emotion regulation across two samples of preschoolers from culturally contrasting countries. We tested whether high levels of effortful control predicted less physiological reactivity and a quicker recovery following a negative emotion-inducing task in both U.S. and Chinese preschool children. We also examined the subcomponents of effortful control, maternal-rated inhibitory control and attentional focusing, together with inhibitory control tested by performancebased tasks, to explore how cognitive determinants of emotion regulation operate across cultures.

Cross-cultural differences in maternal-rated attentional focusing and inhibitory control, as well as the children's performance on the behavioral tasks, were highly consistent with those of previous studies. Namely, Chinese preschool children performed significantly better than their U.S. counterparts on behavioral tasks measuring inhibitory control (Sabbagh et al., 2006). However, U.S. mothers rated their children higher on inhibitory control than Chinese mothers, consistent with data showing that U.S. and Chinese mothers have different perceptions of their children's behavior (Cheah \& Rubin, 2004).

Our study found no cultural differences in the cortisol trajectory itself following the interpersonal stressor. Rather, we found strong cultural differences in the specific subcomponents of EC that predicted cortisol reactivity and recovery, and the direction of the relationship. For U.S. children, we found expected associations between superior maternal-rated inhibitory control, lower cortisol reactivity, and a faster return to baseline following the emotion regulation task. For Chinese children, however, only maternal-rated attentional focusing predicted cortisol reactivity. Furthermore, the latter association was in the opposite direction than expected. Chinese preschoolers whose mothers rated them as having superior levels of attentional focusing skills had higher cortisol reactivity after the emotion regulation task. The beta coefficient for this relation indicated a medium effect size, which held up even after controlling for age, non-verbal intelligence, time between waking and testing, and maternal-rated internalizing problems. Thus, culture appeared to exert a moderating effect on physiological processes of emotion regulation. In what follows, we consider possible explanations for these findings.

First, it is possible that a positive association between attentional focusing and cortisol reactivity in Chinese preschoolers represents a culturally adaptive response to an emotional stressor. Our disappointing prize task was designed to be a strong affective challenge. As stated previously, the socialization of emotion regulation is highly salient in Chinese cultures, even in early childhood (Tardif, Wang, \& Olson, 2009). More specifically, parents in China more rigorously prohibit outward expressions of aggression and are more likely to positively reinforce withdrawn or shy behaviors (Chen, Cen, Li, \& He, 2005; Cheah and Rubin, 2004). Preschools in China more rigorously emphasize order and compliance (Tardif et al., 2009). Moreover, because higher education resources are scarcer, and thus more competitively sought, the expectation of persistence, will power, and fear of failure to achieve begin early in life (Phelps, 2005). In contrast, parents and preschools in Western cultures are more likely to value autonomy in young children and thus be less directive in socializing compliance and suppression of negative affect (Jose et al., 2000; Suizzo et al., 2008; Tardif et al., 2009). It is possible that these cultural emphases exert different types of pressures on emerging regulatory systems. For children growing up in China, exposure to a disappointing event in the presence of an unfamiliar adult may elicit a learned, heightened self-control response and heightened cortisol production due to greater attentional exertion. Our finding that Chinese children showed lower levels of expressed emotions during the challenge compared to U.S. peers, as previously reported elsewhere (Garrett-Peters \& Fox, 2007; Miller et al., 2012), is consistent with this thinking. Indeed, while we found no significant cross-national differences in maternal ratings of attentional focusing, others have shown that elementary school children in China are better able to focus their attention and concentrate in the classroom $(\mathrm{Li}, 2001)$. Thus, a positive association between attentional focusing and physiological reactivity to stress may reflect a normative cultural phenomenon of how children respond in the presence of a potential authority figure.

It is also important to note that U.S. and Chinese children showed the same biological response to the prize task stressor. In other words, we found no mean differences between the two cultures in the rate of the rise and fall of their cortisol levels following the stressor. What we found instead were culturally specific moderators of this biological response. Our findings are consistent with other, albeit sparse, cross-cultural data on biomarkers of emotion regulation. For example, Tsai, Levenson, and McCoy (2006) found that ChineseAmerican and European-American College students did not differ in another physiological measure, cardiac interbeat interval (IBI), during a stressful social task, but the variance in IBI was predicted by level of identification with cultural values. Thus, culture may not directly impact the fundamental functioning of biological mech- 
anisms that help regulate stress and negative emotion, but may influence how cognitive skills and beliefs act on these systems when regulation is required.

Finally, in the U.S. sample, our findings showed specificity as to which subcomponents of effortful control were related to cortisol reactivity and recovery. Although we used multiple behavioral measures and maternal ratings of effortful control, only maternal-rated inhibitory control predicted a more shallow cortisol reactivity and a steeper return to baseline for U.S. preschoolers. Because we had relatively small sample sizes, this may reflect a lack of power to detect effects with our other measures and tasks. Alternatively, this finding may reflect differences across methods of assessment. In the maternal ratings of children's inhibitory control, mothers responded to questions such as "Has difficulty waiting in line for something." These items illustrate examples where broad societal rules are inhibited in the context of high levels of affect, such as excitement or frustration. The performance-based measures, in contrast, were more affectively neutral, and simply required children to understand and inhibit a narrower, novel rule at a more cognitive level. As such, CBQ-Inhibitory Control may measure a more "hot" (affectively salient) version of inhibition, whereas the performance-based tasks of inhibition may measure a more "cool" (affectively neutral) forms of rule suppression involving working memory and lower demands on managing excitement and frustration (Hongwasnishkul, Happaney, Lee, \& Zelazo, 2005). Indeed, Kim and colleagues (2013) found that performance on "hot" tasks of inhibition uniquely predicted preschool children's future externalizing behavior problems, whereas performance on "cool" tasks uniquely predicted future academic achievement, suggesting that the relation between inhibitory control and emotion regulation may be strongly moderated by the level of affective context involved. In addition, lab and parent-based measures of EC also tap into different levels and contexts of the construct. Maternal ratings draw from repeated daily observations of how their children cope with everyday challenges, but with inherent individual and cultural biases about the coping behavior of a "normative" child. Performance-based tasks, in contrast, assess a narrow definition of EC at a single time point but with identical tasks across a variety of children and cultures.

\section{Strengths and Limitations}

The present study had several unique strengths. Examining moment-to moment changes in cortisol, rather than a single-point cortisol sample, enabled us to examine a process model of stress regulation in two cultures. Furthermore, we examined multiple indicators of EC while using identical tasks and measures across cultural groups. This enabled us to uncover a more detailed picture of how cognitive processes tapping into different levels of affect relate to a biological marker of emotion regulation across cultures. Finally, use of path modeling with multi-group analysis enabled us to see how mechanisms of effortful control and cortisol generalized across cultures, rather than just looking at differences in the levels of these variables.

Although our Chinese sample was recruited from suburban areas bordering Beijing, as opposed to the inner city, it is possible that differences in the level of urbanization between the U.S. and Chinese samples contributed to differences in self-regulatory mechanisms such as effortful control and emotion regulation. For example, even within the United States, lower socioeconomic status, higher household density, and fewer parenting resources predict individual differences in effortful control in children and adolescents (Mistry et al., 2004; Compas \& Williams 1990; Evans, Saegert, \& Harrid, 2001). These structural factors have also been related to preschool children's ability to regulate negative emotions (Raver, 2004). Furthermore, Blair and colleagues (2011) found that the relation between economic poverty and early childhood performance on an executive functioning battery measuring working memory, inhibition, and shifting was mediated by individual differences in diurnal cortisol.

We propose, nonetheless, that characteristics such as population density and competition for resources are one aspect of what cultural differences are. In other words, one can think of culture as one manifestation, often over multiple generations, of how socio-demographic factors influence the development of self-regulation skills like effortful control and emotion regulation. Some evidence for thinking about this in the context of cultural change, even in China, also exists (Chen et al., 2005). Regardless of where these values come from initially, differences in the values cultures place on interpersonal functioning are an additional factor in early self-regulation.

In the present study, significant relations between EC subcomponents and cortisol may not have been detected due to lack of power. Specifically, we found an expected negative relation between CBQ IC and cortisol reactivity in the Chinese sample, but this did not reach significance. The sample sizes in the current study were partially determined by challenges in coordinating a complex study protocol in two countries. Replication of the study with larger samples may reveal additional significant pathways and cultural differences. Alternatively, the reduced reliability of the CBQ- IC scale in the Chinese 
sample may have affected our findings. Clearly, future research is needed to disentangle these effects.

Based on our findings, which suggest that there are differences in how different components of EC impact physiological processes of emotion regulation in young children, we strongly recommend that future research examining culture, effortful control, and emotion regulation use constructs with multiple indicators. A push towards examining effortful control and emotion regulation skills at different levels and contexts (Cole, Martin, and Dennis, 2004) has not adequately made its way to cross-cultural studies of children. In the current study, treating subcomponents of effortful control separately provided a more complex picture of cultural differences than a single indicator or composite would have.

We also recommend that future cross-cultural research utilize analyses capable of examining how processes generalize across cultures. Future research using similar methodologies can test whether other complex theoretical models developed from data on Western children replicate in non-Western samples, and provide an important, much deeper understanding of culture and development. We encourage future cross-cultural research to test other models of emotion regulation with different theoretical pathways.

\section{Implications}

Our findings suggest that individuals raised in societies with culturally-specific differences in parenting practices, educational institutions, and broader societal norms may develop different patterns of emotion regulation competence. The degree to which a society tolerates or reinforces emotional behavior and expression may shape how cognitive skills and neuroendocrine systems work together during affectively negative events. The current study is the first to suggest that emerging emotion regulation systems appear to be impacted by culture both very early in childhood and at a physiological level.

\section{Acknowledgements}

This research was funded by NSF grant SES 0527475 to Twila Tardif. In addition, we would like to thank the children, families, and preschools that participated in our research in both China and the U.S., as well as the numerous research assistants and collaborators who made this research possible.

\section{References}

Achenbach, T.M., \& Rescorla, L.A. (2000). Manual of ASEBA preschool forms and profiles. Burlington, VT: University of Vermont, Research Center for Children, Youth and Families.

Ahadi, S.A., Rothbart, M.K., \& Ye, R. (1993). Children's temperament in the U.S. and China: similarities and differences. European Journal of Personality, 7, 359-377. doi:10. 1002/per.2410070506

Beauchaine, T.P. (2009). Some difficulties in interpreting psychophysiological research with children. Monographs of the Society for Research in Child Development, 74 (1), 80-88. doi:10.1111/j.1540-5834.2009.00509.x

Best, J.R., \& Miller, P.H. (2010). A developmental perspective on executive function. Child Development, 81 (6), 1641-1660. doi: 10.1111/j.1467-8624.2010.01499.x

Blair, C., Granger, D., \& Razza, R.P. (2005). Cortisol reactivity is positively related to executive function in preschool children attending head start. Child Development, 76 (3), 554-567. doi: 10.1111/j.1467-8624.2005.00863.x

Blair, C., Granger, D.A., Willoughby, M., Mills-Koonce, R., Cox, M., Greenberg, M.T., Kivlighan, K.T., \& Fortunato, C.K. (2011). Salivary cortisol mediates effects of poverty and parenting on executive functions in early childhood. Child Development, 82 (6), 1970-1984. doi: 10.1111/j.1467-8624. 2011.01643.x

Buckley, T.M., \& Schatzberg, A.F. (2005). On the interactions of the hypothalamic-pituitary-adrenal (HPA) axis and sleep: normal HPA axis activity and circadian rhythm, exemplary sleep disorders. Journal of Clinical Endocrinology \& Metabolism, 90 (5), 3106-3114. doi: 10.1210/jc.20041056

Calkins, S., \& Hill, A. (2007). Caregiver influences on emerging emotion regulation. Handbook of emotion regulation, 229248.

Carlson, S.M., \& Moses, L.J. (2001). Individual differences in inhibitory control and children's theory of mind. Child Development, 72 (4), 1032-1053. doi: 10.1111/1467-8624. 00333

Chao, R.K. (1994). Beyond parental control and authoritarian parenting style: Understanding Chinese parenting through the cultural notion of training. Child Development, 65 (4), 1111-1119. doi: 10.1111/j.1467-8624.1994.tb00806.x

Chao, R.K. (2001). Extending research on the consequences of parenting style for Chinese Americans and European Americans. Child Development, 72 (6), 1832-1843. doi: 10.1111/ 1467-8624.00381

Cheah, C.L., \& Rubin, K.H. (2004). European American and Mainland Chinese mothers' responses to aggression and social withdrawal in preschoolers. International Journal of Behavioral Development, 200 (28), 83-94. doi: 10.1080/ 01650250344000299

Chen, X., Cen, G., Li, D., \& He, Y. (2005). Social functioning and adjustment in Chinese children: the imprint of historical time. Child Development, 76 (1), 182-195. doi: 10.1111/j. 1467-8624.2005.00838.x 
Cole, P.M. (1986). Children's spontaneous control of facial expression. Child Development, 57 (6), 1309-1321. doi: 10. 2307/113041

Cole, P.M., Martin, S.E., \& Dennis, T.A. (2004). Emotion regulation as a scientific construct: methodological challenges and directions for child development research. Child Development, 75 (2), 317-333. doi: 10.1111/j.1467-8624.2004. 00673.x

Compas, B.E., \& Williams, R.A. (1990). Stress, coping, and adjustment in mothers and young adolescents in single-and two-parent families. American Journal of Community Psychology, 18 (4), 525-545. doi: 10.1007/BF00938058 10.1007/ BF00938058

De Haan, M., Gunnar, M.R., Tout, K., Hart, J., \& Stansbury, K. (1998). Familiar and novel contexts yield different associations between cortisol and behavior among 2-yearold children. Developmental Psychobiology, 33 (1), 93-101. doi: 10.1002/(SICI)1098-2302(199807)33:1<93:AIDDEV8>3.0.CO;2-N

Dettling, A.C., Gunnar, M.R., \& Donzella, B. (1999). Cortisol levels of young children in full-day childcare centers: Relations with age and temperament. Psychoneuroendocrinology, 24 (5), 519-536. doi: 10.1016/S0306-4530 (99)00009-8

Diamond, A., \& Taylor, C. (1996). Development of an aspect of executive control: Development of the abilities to remember what I said and to "Do as I say, not as I do". Developmental Psychobiology, 29 (4), 315-334. doi: 10.1002/(SICI)1098-2302 (199605)29:4<315:AID-DEV2>3.0.CO;2-T

Dickerson, S.S., \& Kemeny, M.E. (2004). Acute stressors and cortisol responses: a theoretical integration and synthesis of laboratory research. Psychological Bulletin, 130 (3), 355-391. doi: 10.1037/0033-2909.130.3.355

Earle, T.L., Linden, W., \& Weinberg, J. (1999). Differential effects of harassment on cardiovascular and salivary cortisol stress reactivity and recovery in women and men. Journal of psychosomatic research, 46 (2), 125-141. doi: 10.1016/S00223999(98)00075-0

Eisenberg, N., Chang, L., Ma, Y., \& Huang, X. (2009). Relations of parenting style to Chinese children's effortful control, ego resilience, and maladjustment. Development and psychopathology, $21 \quad$ (2), 455 . doi: $10.1017 /$ S095457940900025X

Eisenberg, N., Ma, Y., Chang, L., Zhou, Q., West, S.G., \& Aiken, L. (2007). Relations of effortful control, reactive undercontrol, and anger to Chinese children's adjustment. Development and Psychopathology, 19 (02), 385-409. doi: 10. 1017/S0954579407070198

Essex, M.J., Shirtcliff, E.A., Burk, L.R., Ruttle, P.L., Klein, M.H., Slattery, M.J., Kalin, N.H., \& Armstrong, J.M. (2011). Influence of early life stress on later hypothalamic-pituitaryadrenal axis functioning and its covariation with mental health symptoms: a study of the allostatic process from childhood into adolescence. Development and psychopathology, 23 (4), 1039. doi: 10.1017/S0954579411000484

Evans, G. W., Saegert, S., \& Harris, R. (2001). Residential density and psychological health among children in low-income families. Environment and Behavior, 33(2), 165180. doi: $10.1177 / 00139160121972936$

Garavan, H., Ross, T.J., \& Stein, E.A. (1999). Right hemispheric dominance of inhibitory control: an event-related functional MRI study. Proceedings of the National Academy of Sciences, 96 (14), 8301-8306. doi: 10.1073/pnas.96.14. 8301

Garrett-Peters, P.T., \& Fox, N.A. (2007). Cross-cultural differences in children's emotional reactions to a disappointing situation. International Journal of Behavioral Development, 31, 161-169. doi: 10.1177/0165025407074627

Gross, J. J. (1998). The emerging field of emotion regulation: An integrative review. Review of general psychology, 2 (3), 271. doi: 10.1037/1089-2680.2.3.271

Gross, J. J. (Ed.). (2009). Handbook of emotion regulation. Guilford Press.

Gunnar, M.R., \& Donzella, B. (2002). Social regulation of the cortisol levels in early human development. Psychoneuroendocrinology, 27 (1), 199-220. doi: 10.1016/S0306-4530(01) 00045-2

Gunnar, M.R., Sebanc, A.M., Tout, K., Donzella, B., \& van Dulmen, M.M. (2003). Peer rejection, temperament, and cortisol activity in preschoolers. Developmental psychobiology, 43 (4), 346-368. doi: 10.1002/dev.10144

Gunnar, M.R., \& Vazquez, D.M. (2001). Low cortisol and a flattening of expected daytime rhythm: potential indices of risk in human development. Development and Psychopathology, 13 (3), 515-538. doi: 10.1017/S0954579401003066

Gunnar, M. R., \& Vazquez, D. (2006). Stress neurobiology and developmental psychopathology. In D. Cicchetti \& D. Cohen (Eds.), Developmental psychopathology: Vol. III. Risk, disorder, and adaptation (2nd ed., pp. 533-577). New York, NY: Wiley.

Hongwanishkul, D., Happaney, K.R., Lee, W.S.C., \& Zelazo, P.D. (2005). Assessment of hot and cool executive function in young children: age related changes and individual differences. Developmental Neuropsychology, 28, 617-644. doi: 10. $1207 / \mathrm{s} 15326942 \mathrm{dn} 28024$

Ingram, R.E. (1990). Self-focused attention in clinical disorders: review and a conceptual model. Psychological Bulletin, 107 (2), 156-176. doi: 10.1037/0033-2909.107.2.156

Izard, C.E. (2009). Emotion theory and research: Highlights, unanswered questions, and emerging issues. Annual review of psychology, 60, 1. doi: 10.1146/annurev.psych.60.110707. 163539

Jose, P.E., Huntsinger, C.S., Huntsinger, P.R., \& Liaw, F. (2000). Parental values and practices relevant to young children's social development in Taiwan and the United States. Journal Of Cross-Cultural Psychology, 31 (6), 677-702. doi: 10.1177/0022022100031006002

Kagan, J., Reznick, J.S., \& Snidman, N. (1988). Biological bases of childhood shyness. Science, 240, 167-171. doi: 10.1126/ science. 3353713

Kim, S., Nordling, J.K., Yoon, J.E., Boldt, L.J., \& Kochanska, G. (2013). Effortful Control in "Hot" and "Cool" Tasks Differentially Predicts Children's Behavior Problems and Academic Performance. Journal of abnormal child psychology, 41 (1), 43-56. doi: 10.1007/s10802-012-9661-4 
Kochanska, G., Murray, K.T., \& Harlan, E.T. (2000). Effortful control in early childhood: continuity and change, antecedents, and implications for social development. Developmental Psychology, 36 (2), 220-232. doi: 10.1037/0012-1649.36.2.220

Kochanska, G., Murray, K.T., Jacques, T.Y., \& Vandegeest, K.A. (1996). Inhibitory control in young children and its role in emerging internalization. Child Development, 67 (2), 490507. doi: 10.1111/j.1467-8624.1996.tb01747.x

Kopp, C.B. (1989). Regulation of distress and negative emotions: A developmental view. Developmental Psychology, 25 (3), 343. doi: 10.1037/0012-1649.25.3.343

Lan, X., Legare, C.H., Ponitz, C.C., Li, S., \& Morrison, F.J. (2011). Investigating the links between the subcomponents of executive function and academic achievement: A crosscultural analysis of Chinese and American preschoolers. Journal of Experimental Child Psychology, 108 (3), 677-692. doi: 10.1016/j.jecp.2010.11.001

Li, J. (2001). Chinese conceptualization of learning. Ethos, 29 (2), 111-137. doi: 10.1111/j.1467-8624.1996.tb01747.x

Liu, M., Chen, X., Rubin, K.H., Zheng, S., Cui, L., Li, D., Chen, H., \& Wang, L. (2005). Autonomy- vs. connectednessoriented parenting behaviours in Chinese and Canadian mothers. International Journal of Behavioral Development, 29 (6), 489-495. doi: 10.1080/01650250500147063

Lopez-Duran, N.L., Olson, S.L., Hajal, N.J., Felt, B.T., \& Vazquez, D.M. (2009). Hypothalamic pituitary adrenal axis functioning in reactive and proactive aggression in children. Journal of Abnormal Child Psychology, 37 (2), 169-182. doi: 10.1007/s10802-008-9263-3

Luijk, M.P., Saridjan, N., Tharner, A., van Ijzendoorn, M.H., Bakermans-Kranenburg, M.J., Jaddoe, V.W.V., Hofman, A., Verhulst, F.C., \& Tiemeier, H. (2010). Attachment, depression, and cortisol: Deviant patterns in insecure-resistant and disorganized infants. Developmental Psychobiology, 52 (5), 441-452. doi: 10.1002/dev.20446

Luria, A.R., Pribram, K.H., \& Homskaya, E.D. (1964). An experimental analysis of the behavioral disturbance produced by a left frontal arachnoidal endothelioma (meningioma). Neuropsychologia, 2, 257-280. doi: 10.1016/0028-3932(64) 90034-X

Martin, M., Horder, P., \& Jones, G.V. (1992). Integral bias in naming of phobia-related words. Cognition \& Emotion, 6, 479-486. doi: 10.1080/02699939208409700

Miller, A. L., Lee, H., Karasawa, M., Wang, L., Kessler, D., Tardif, T. Z., Olson, S. L., \& Hirabayashi, H. (2012). Facial expressions of emotion in three cultures. Symposium presented at the Meeting for the International Society for the Study of Behavioural Development, Edmonton, AB (July, 2012).

Mistry, R.S., Biesanz, J.C., Taylor, L.C., Burchinal, M., \& Cox, M.J. (2004). Family income and its relation to preschool children's adjustment for families in the NICHD Study of Early Child Care. Developmental psychology, 40 (5), 727. doi: 10.1037/0012-1649.40.5.727

Muthén, L.K., \& Muthén, B.O. (1998). MPlus. Los Angeles: Stat Model.

Olson, S.L., Sameroff, A.J., Kerr, D.C., Lopez, N.L., \& Wellman, H.L. (2005). Developmental foundations of externalizing problems in young children: The role of effortful control. Development and Psychopathology, 17 (1), 25-45. doi: 10.1017/S0954579405050029

Olson, S.L., Tardif, T.Z., Miller, A., Felt, B., Grabell, A.S., Kessler, D., Li, W., Karasawa, M., \& Hirabayashi, H. (2011). Inhibitory control and harsh discipline as predictors of externalizing problems in young children: a comparative study of US, Chinese, and Japanese preschoolers. Journal of Abnormal Child Psychology, 39 (8), 1163-1175. doi: 10.1007/ s10802-011-9531-5

Phelps, L. (2005). Academic achievement of children in China: The 2002 Fulbright experience. Psychology in the Schools, 42 (3), 233-239. doi: 10.1002/pits.20074

Posner, M.I., \& Rothbart, M.K. (2000). Hebb's neural networks support the integration of psychological science. Canadian Psychology, 45 (4), 265-278. doi: 10.1037/ h0086997

Raghunathan, T. E., Solenberger, P. W., \& Van Hoewyk, J. (2002). Imputation and variance estimation software users guide. Ann Arbor, MI: Survey Methodology Program, Survey Research Center, Institute for Survey Research, University of Michigan.

Raver, C.C. (2004). Placing Emotional Self-Regulation in Sociocultural and Socioeconomic Contexts. Child Development, 75 (2), 346-353. doi: 10.1111/j.1467-8624.2004.00676.x

Rothbart, M.K. (1989). Temperament and development. In G.A. Kohnstamm, J.E. Bates \& M.K. Rothbart (Eds.), Temperament in childhood (pp. 187-247). New York: Wiley.

Rothbart, M.K., Ahadi, S.A., Hershey, K.L., \& Fisher, P. (2000). Investigations of temperament at three to seven years: the children's behavior questionnaire. Child Development, 72 (5), 1394-1408. doi: 10.1111/1467-8624.00355

Rothbart, M. K., \& Rueda, R. M. (2005). The development of effortful control. In U. Mayer, E. Awh \& S.W. Keele (Eds.), Developing Individuality in the Human Brain: A Tribute to Michael I. Posner(pp. 167-188). Washington, DC: American Psychological Association.

Saarni, C. (1984). An observational study of children's attempts to monitor their expressive behavior. Child Development, 55 (4), 1504-1513. doi: 10.2307/1130020

Sabbagh, M.A., Xu, F., Carlson, S.M., Moses, L.J., \& Lee, K. (2006). The development of executive functioning and theory of mind: a comparison of Chinese and U.S. preschoolers. Psychological Science, 17 (1), 74-81. doi: 10.1111/j.14679280.2005.01667.x

Schmidt-Reinwald, A., Pruessner, J.C., Hellhammer, D.H., Federenko, I., Rohleder, N., Shürmeyer, T.H., \& Kirschbaum, C. (1999). The cortisol response to awakening in relation to different challenge tests and a 12-hour cortisol rhythm. Life Sciences, 64 (18), 1653-1660. doi: 10.1016/ S0024-3205(99)00103-4

Spinrad, T.L., Eisenberg, N., Granger, D.A., Eggum, N.D., Sallquist, J., Haugen, R.G., Kupfer, A., \& Hofer, C. (2009). Individual differences in preschoolers' salivary cortisol and alpha-amylase reactivity: reactions to temperament and maladjustment. Hormones and Behavior, 56 (1), 133-139. doi: 10.1016/j.yhbeh.2009.03.020 
Suizzo, M., Chen, W., Cheng, C., Liang, A.S., Contreras, H., Zanger, D., \& Robinson, C. (2008). Parental beliefs about young children's socialization across US ethnic groups: Coexistance of independence and interdependence. Early Child Development and Care, 178 (5), 467-486. doi:10.1080/ 03004430600823917. doi:10.1080/03004430600823917

Sullivan, R.M. (2004). Hemispheric asymmetry in stress processing in rat prefrontal cortex and the role of mesocortical dopamine. Stress: The International Journal on the Biology of Stress, 7 (2), 131-143. doi: 10.1080/ 102538900410001679310

Tardif, T., So, C., \& Kaciroti, N. (2007). Language and false belief: Evidence for general, not specific, effects in Cantonese-speaking preschoolers. Developmental Psychology, 43 (2), 318-340. doi: 10.1037/0012-1649.43.2.318

Tardif, T., Wang, L., \& Olson, S. L. (2009). Culture and the development of regulatory competence: Chinese-U.S. comparisons. In Olson, S. L. , Sameroff \& A. J. (Eds.) Biopsychosocial regulatory processes in the development of childhood behavioral problems (pp. 258-289). New York: Cambridge University Press.

Tsai, J.L., Levenson, R.W., \& McCoy, K. (2006). Cultural and temperamental variation in emotional response. Emotion, 6 (3), 484-497. doi: 10.1037/1528-3542.6.3.484

Thompson, R.A. (1994). Emotion regulation: A theme in search of definition. Monographs of the society for research in child development, 59 (2-3), 25-52. doi: 10.1111/j.1540-5834. 1994.tb01276.x

Thompson, R.A. (2011). Emotion and emotion regulation: Two sides of the developing coin. Emotion Review, 3 (1), 53 61. doi: $10.1177 / 1754073910380969$
Ulrich-Lai, Y.M., \& Herman, J.P. (2009). Neural regulation of endocrine and autonomic stress responses. Nature Reviews Neuroscience, 10 (6), 397-409. doi: 10.1038/nrn2647

Vasey, M.W., Daleiden, E.L., Williams, L.L., \& Brown, L.M. (1995). Biased attention in childhood anxiety disorders: A preliminary study. Journal of Abnormal Child Psychology, 23, 267-279. doi: 10.1007/BF01447092

Wang, L., Chen, X., Chen, H., Cui, L., \& Li, M. (2006). Affect and maternal parenting as predictors of adaptive and maladaptive behaviors in Chinese children. International Journal of Behavioral Development, 30 (2), 158-166. doi: 10. 1177/0165025406063631

Wechsler, D. (2002). WPPSI-III Administration and Scoring Manual. San Antonio, TX: Psychological Corporation.

Xu, Y., Farver, J.A.M., Zhang, Z., Zeng, Q., Yu, L., \& Cai, B. (2005). Mainland Chinese parenting styles and parent-child interaction. International Journal of Behavioral Development, 29 (6), 524-531. doi: 10.1177/01650250500147121

Zelazo, P.D., Müller, U., Frye, D., \& Marcovitch, S. (2003). The development of executive function in early childhood: 1 . the development of executive function. Monographs of the Society for Research in child Development, 68 (3), 11-27. doi: 10.1037/1089-2680.1.2.198

Zhou, Q., Lengua, L.J., \& Wang, Y. (2009). The relations of temperament reactivity and effortful control to children's adjustment problems in China and the United States. Developmental psychology, 45 (3), 724. doi: 10.1037/ a0013776

Received: 21 February 2013

Accepted: 17 June 2014 